\title{
The $\mu$-RWELL: a compact, spark protected, single amplification-stage MPGD
}

\section{G. Bencivenni* Laboratori Nazionali di Frascati - INFN, Frascati, Italy}

E-mail: giovanni.bencivennielnf.infn.it

M. Gatta, G. Morello, M. Poli Lener

Laboratori Nazionali di Frascati - INFN, Frascati, Italy

\section{R. De Oliveira}

CERN, Meyrin, Switzerland

By combining in a unique approach the solutions and improvements realized in the last years in the Micro-pattern-gas-detector (MPGD) field, we have developed a novel detector architecture called micro-Resistive-WELL ( $\mu$-RWELL): a compact spark-protected single amplification stage MPGD. The amplification stage of the detector, realized with a structure very similar to a GEM foil, is embedded through a resistive layer with the readout board. A cathode electrode, defining the gas conversion-drift gap, complete the detector mechanics.

The tests performed on small prototypes, with different resistivity from $80 \mathrm{M} \Omega$ /square to $100 \mathrm{M} \Omega /$ square have shown that the new structure exhibits a gain of more than $10^{4}$, while the discharges are strongly suppressed thanks to the presence of the resistive layer. A rate capability of about $600 \mathrm{kHz} / \mathrm{cm}^{2}$ at a gain of 5000 has been measured. Preliminary results from a beam test with the $80 \mathrm{M} \Omega /$ square prototype, equipped with a $400 \mu \mathrm{m}$ pitch strip readout, show a space resolution better than $60 \mu \mathrm{m}$ with a track reconstruction efficiency of $\sim 98 \%$ (with $4 \mathrm{~mm}$ gas gap).

International Winter Meeting on Nuclear Physics,

26-30 January 2015

Bormio, Italy

\footnotetext{
* Speaker.
} 


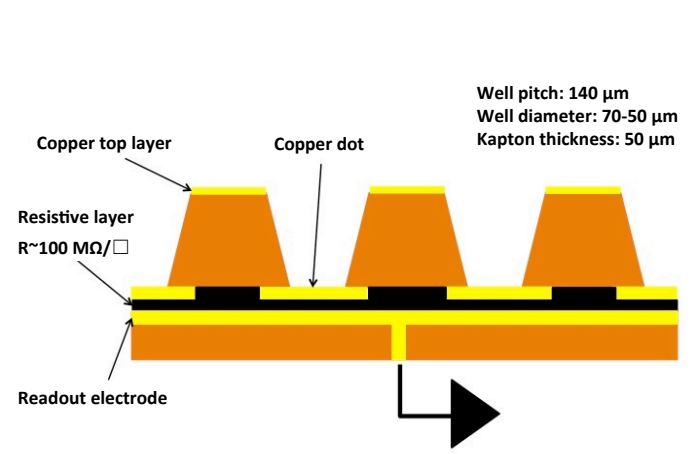

Figure 1: Schematic drawing of the $\mu$-RWELL PCB.

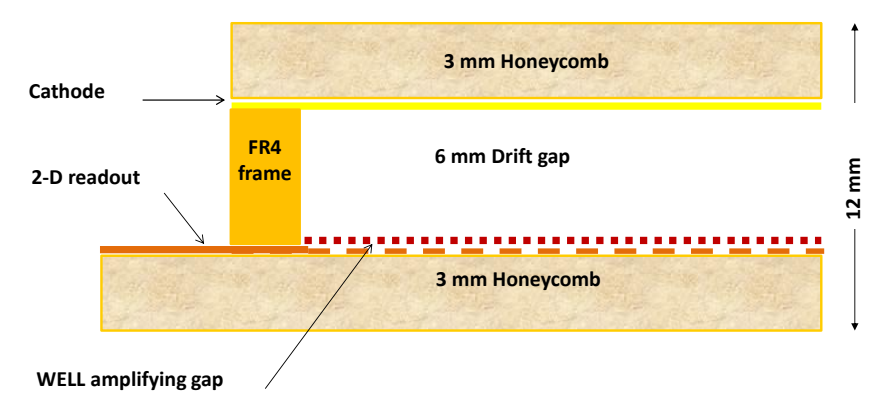

Figure 2: Schematic drawing of the $\mu$-RWELL detector.

\section{Detector description}

The $\mu$-RWELL [1] has been designed at the Laboratori Nazionali di Frascati and realized the first time in the 2009 by TE-MPE-EM Workshop at CERN in parallel with the CERN-GDD group $[2,3]$. A similar device based on THGEM technology has been recently proposed by other groups [4].

The $\mu$-RWELL, as sketched in fig. 1 , is realized by merging a suitable etched GEM foil with the readout PCB plane coated with a resistive deposition. The copper on the bottom side of the foil has been patterned in order to create small metallic dots in correspondence of each WELL structure. The resistive coating has been performed by screen printing technique. The WELL matrix geometry is realized on a $50 \mu \mathrm{m}$ thick polyimide foil, with conical channels $70 \mu \mathrm{m}(50 \mu \mathrm{m})$ top (bottom) diameter and $140 \mu \mathrm{m}$ pitch (of course different geometries can be considered in order to optimize the detector performance, especially in terms of gain amplitude). A cathode electrode, defining the gas conversion/drift gap, completes the detector mechanics (fig. 2).

The $\mu$-RWELL has features in common either with GEMs [5] or Micromegas [6]:

- from GEM it takes the amplifying scheme with the peculiarity of a "well defined amplifying gap", thus ensuring very high gain uniformity. While Micromegas, using "floating meshes", have lost this property moving to large area.

- from Micromegas it takes the resistive readout scheme that allows a strong suppression of the amplitude of the discharges. The principle is the same of the resistive electrode used in the RPCs $[7,8,9]$.

Even though the amplifying element of the $\mu$-RWELL is practically the same of the GEM, its signal formation mechanism is completely different. The signal in a GEM detector is mainly due to the electron motion, while in a $\mu$-RWELL besides the very fast collection of the whole electron charge produced into the amplification channel also the ionic component, apart ballistic effects correlated 


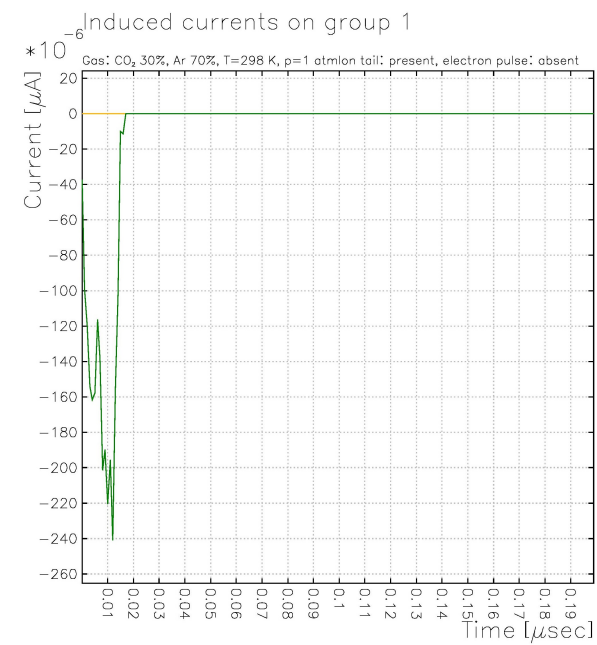

Figure 3: GARFIELD simulation of a signal from a single ionization electron in a single-GEM. The duration of the signal, about $20 \mathrm{~ns}$, depends on the induction gap geometry, drift velocity and fields in the gap.

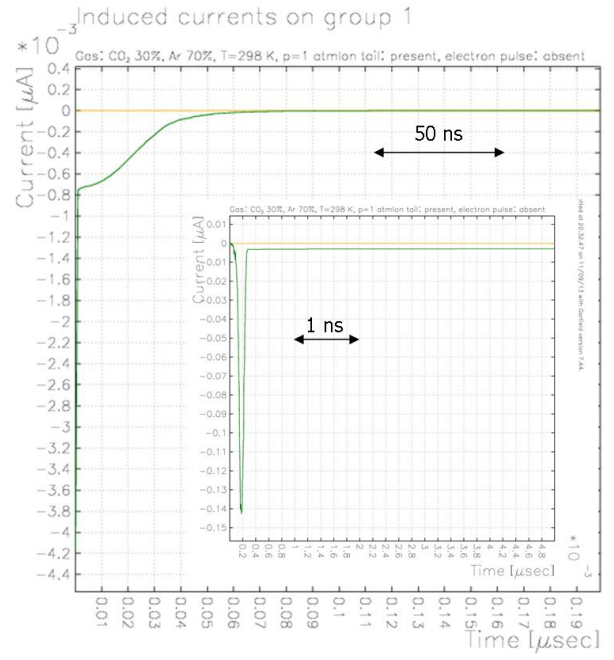

Figure 4: GARFIELD simulation of a signal from a single ionization in a $\mu$-RWELL. The fast initial spike, about $200 \mathrm{ps,} \mathrm{induced} \mathrm{by} \mathrm{the} \mathrm{motion}$ and fast collection of electrons is followed by a 50 ns ion tail.

with the integration time of the readout electronics, contributes to the formation of the signal. In this sense the signal of a resistive-WELL is more similar to the one of a MM. In fig. 3 and fig. 4 a comparison between the simulation of the signal generated by a single ionization electron in a single-GEM and a R-WELL is reported $\left(\mathrm{Ar} / \mathrm{CO}_{2}=70 / 30\right.$ gas mixture).

The assembly aspect of the resistive-WELL technology is obviously a strong point in favor of this architecture. The $\mu$-RWELL is composed by only two components: the readout-PCB, with the amplifying part embedded on it, and the cathode. Its assembly does not require gluing or stretching of foils or meshes: a very critical and time-consuming construction step of both GEM and MM technologies. The stretching of a GEM foil as well as a metallic mesh requires mechanical tension (of the order of $1 \mathrm{~kg} / \mathrm{cm}$ along the perimeter of the surface to be stretched), that clearly must be supported by suitable rigid mechanical structures.

The $\mu$-RWELL with respect to the GEM and MM is extremely compact, does not require very stiff (and large) support structures, allowing large area covering based on a PCB-splicing technique (with a dead space within $0.2 \div 0.3 \mathrm{~mm}$ ) while keeping a "well defined amplifying gap".

\section{Detector performance}

In the following we report the results about the tests performed on the $\mu$-RWELL prototypes. The detector gain has been measured with X-rays, in current mode, as a function of the potential applied across the amplification stage and the resistive layer. As shown in fig. 5, the operation with an iso-buthane based gas mixture allows to achieve a gas gain larger than $10^{4}$, comparable with the gain at which standard triple-GEM and MM are normally operated. The use of thicker kapton foil $(125 \mu \mathrm{m}$ thick polyimide foil is commercially available, from KANEKA ltd in Japan) for the 


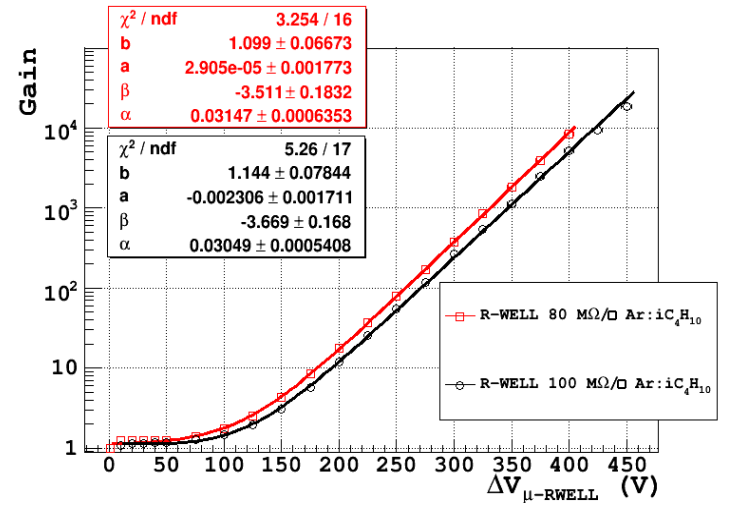

Figure 5: Gas gain in Ar:iso-buthane (90:10), for two different $\mu$-RWELL geometry: $80 \mathrm{M} \Omega$ /square (red points) and $100 \mathrm{M} \Omega /$ square (black points).

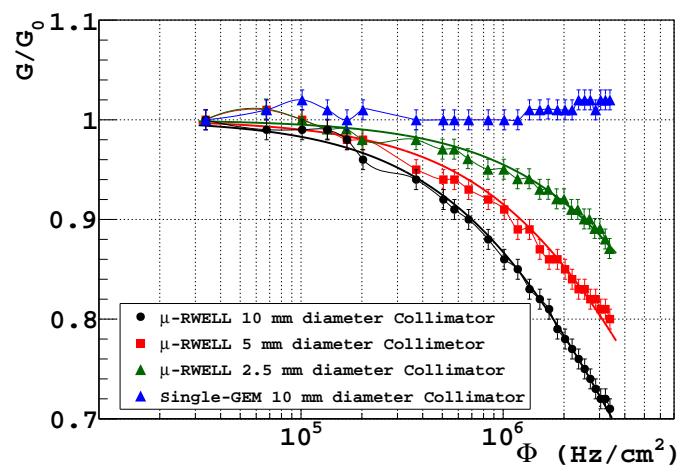

Figure 6: Comparison of the normalized gain (a.u.) for the GEM (blue) and the $\mu$-RWELL for different collimator diameters $\left(\mathrm{G}_{0}=3000\right.$ with $\mathrm{Ar}: \mathrm{CO}_{2}$ 70:30).

realization of the amplifying component of the detector should allow to achieve gas gain sensibly larger than those obtained with the $50 \mu \mathrm{m}$ thick amplification gap.

The introduction of a high resistivity layer between the amplification stage and the readout reduce the capability to stand very high particle fluxes. The results, fig. 6, indicate that the rate capability of the detector can be tuned (tipically from $100 \mathrm{kHz} / \mathrm{cm}^{2}$ to $600 \mathrm{kHz} / \mathrm{cm}^{2}$, for a surface resistivity of about $100 \mathrm{M} \Omega$ /square) with a suitable segmentation of the resistive layer [4].

The typical discharge amplitude for the $\mu$-RWELL is of the order of few tens of nA, while

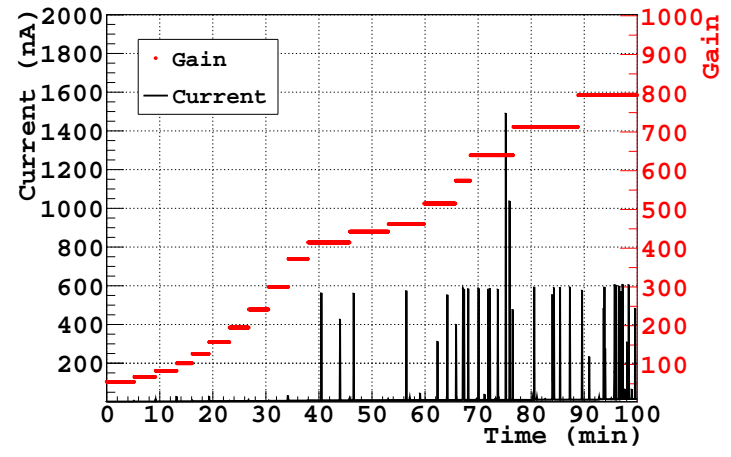

Figure 7: Monitoring of the current drawn (in black) by the single-GEM detector for different gas gain (in red).

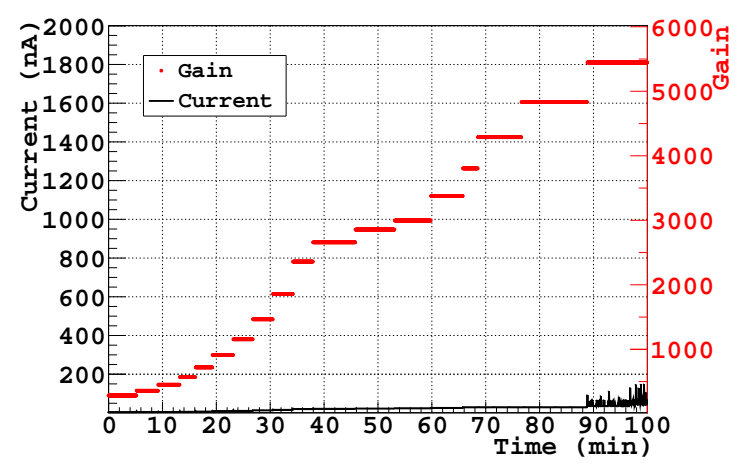

Figure 8: Monitoring of the current drawn (in black) by the $\mu$-RWELL detector for different gas gain (in red).

for a GEM detector discharges with amplitude of the order of $\mu \mathrm{A}$ are observed at high gas gain (fig. 7 and fig. 8). Preliminary results from a test at the H4-SPS beam line with a $80 \mathrm{M} \Omega$ /square $\mu$-RWELL equipped with a $400 \mu \mathrm{m}$ pitch strip readout (coupled with APV25 chip boards, allowing center of gravity method - COG) show a space resolution down to $54 \pm 2 \mu \mathrm{m}$ (after the subtraction of the contribution due to the track fit, $42 \pm 2 \mu \mathrm{m}$, performed with three triple-GEM detectors) fig. 9 , with a detection efficiency of the order of $\sim 98 \%$ (with 4 mm gas gap) fig. 10 . 


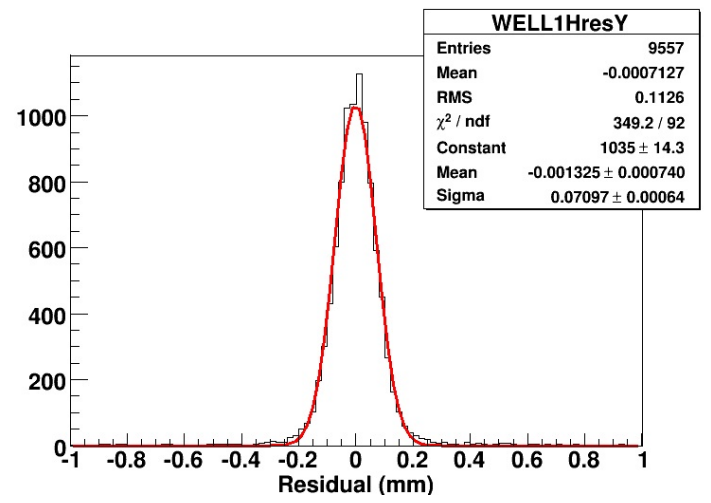

Figure 9: Track fit residual for the $\mu$-RWELL for orthogonal incidence and $\mathrm{B}=0$ Tesla. The estimated space resolution of the $\mu$-RWELL is $54 \pm 2 \mu \mathrm{m}$.

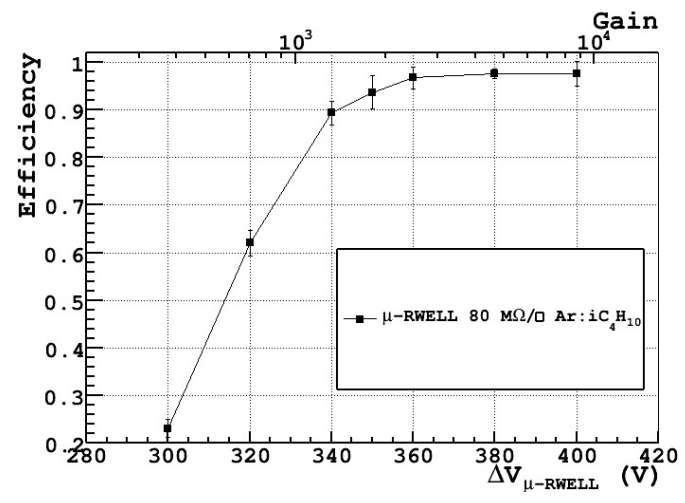

Figure 10: Track reconstruction efficiency for $\mu$ RWELL, with 1D strip readout, for othogonal incidence and $\mathrm{B}=0$ Tesla .

\section{Conclusions}

The $\mu$-RWELL is a very promising technology showing important advantages with respect to classical GEMs and MMs: the detector is "thin - large - simple" and exhibits effective spark quenching. Besides high gas gain $\left(>10^{4}\right)$ and good rate capability $\left(\sim 600 \mathrm{kHz} / \mathrm{cm}^{2}\right.$ at $\mathrm{G} \sim 5000$ and a resistivity of about $100 \mathrm{M} \Omega /$ square - without resistive layer segmentation) a space resolution better than $60 \mu \mathrm{m}$ has been measured.

\section{References}

[1] G. Bencivenni at al., The micro-Resistive WELL detector: a compact spark-protected single amplification-stage MPGD, JINST 10 (2015) P02008.

[2] M. Alfonsi et al., Performance measurements on closed-geometry GEM like detectors, presented at the MPGD2009 - 1st International Conference on Micropattern Gaseous Detectors, 12-15 June 2009 Crete.

[3] G. Croci, Development and characterization of Micro-Pattern Gaseous Detectors for HEP applications and beyond, Ph.D Thesis, XXIII cycle (2010), University of Siena.

[4] L. Arazi et al., Beam studies of the Segmented Resistive WELL: a potential thin sampling element for Digital Hadronic Calorimetry, Nucl. Inst. \& Meth. A 732 (2013) 199.

[5] F. Sauli, GEM: A new concept for electron amplification in gas detectors., Nucl. Inst. \& Meth. A 386 (1997) 531.

[6] I. Giomataris et al., Micromegas: a high-granularity, position sensitive gaseous detector for high particle flux environments., Nucl. Inst. \& Meth. A 376 (1996) 29.

[7] Y. Pestov et al., A spark counter with large area, Nucl. Instr. \& Meth. 93 (1971) 269.

[8] R. Santonico, R. Cardarelli, Development of Resistive Plate Counters, Nucl. Instr. \& Meth. A 377 (1981) 187.

[9] M. Anelli et al., Glass electrode spark counters, Nucl. Instr. \& Meth. A 300 (1991) 5072.

[10] G. Bencivenni, R. De Oliveira, Private communication (2014). 